\title{
Acute reperfusion intramyocardial hemorrhage leads to regional chronic iron deposition in the heart
}

\author{
Avinash Kali ${ }^{1}{ }^{2}$, Ivan Cokic ${ }^{1}$, Andreas Kumar ${ }^{3}$, Sotirios Tsaftaris ${ }^{4}$, Richard L Tang ${ }^{1}$, Matthias G Friedrich ${ }^{5,6}$, \\ Rohan Dharmakumar' \\ From 16th Annual SCMR Scientific Sessions \\ San Francisco, CA, USA. 31 January - 3 February 2013
}

\section{Background}

Intramyocardial hemorrhage commonly occurs in large reperfused myocardial infarctions. However, its long-term fate remains unexplored. We hypothesized that acute reperfusion intramyocardial hemorrhage leads to chronic iron deposition.

\section{Methods}

Fifteen patients (mean age $=58 \pm 8$ years; 3 women), who underwent successful angioplasty for first STEMI, were recruited following informed consent. Cardiovascular Magnetic Resonance (CMR) imaging (1.5T) was performed on day 3 and month 6 post-angioplasty. 2D T2* maps $(6$ $\mathrm{TEs}=2.6-13.7 \mathrm{~ms} ; \Delta \mathrm{TE}=2.2 \mathrm{~ms}$ ) and Late Gadolinium Enhancement (LGE) images of the entire left ventricle (LV) were acquired. Threshold-based image analysis was performed to identify remote, hemorrhagic $(\mathrm{Hemo}+)$ and non-hemorrhagic (Hemo-) myocardium.

Fourteen canines, subjected to ischemia-reperfusion (I-R) injury (3 hours of LAD occlusion followed by reperfusion), underwent CMR (1.5T) on days 3 and 56 post-I-R injury. Three sham-operated animals (Shams) were also studied using CMR at similar time points. $2 \mathrm{D}$ T2* maps $(6 \mathrm{TEs}=$ 3.4-18.4 ms; $\Delta \mathrm{TE}=3.0 \mathrm{~ms}$ ) and LGE images of the entire LV were acquired. Threshold-based image analysis was performed to identify remote, Hemo+ and Hemo- myocardium. Subsequently, animals were euthanized (day 56), hearts were excised and imaged ex-vivo. Sections of Hemo +, Hemo-, remote and Sham myocardium were isolated and histology was performed. The concentration of iron

'Biomedical Imaging Research Institute, Cedars-Sinai Medical Center, Los Angeles, CA, USA

Full list of author information is available at the end of the article
([Fe]) within each type of tissue was measured using mass spectrometry.

\section{Results}

Six months post-angioplasty, mean $\mathrm{T} 2 *$ of the scar tissue in patients with Hemo+ infarctions ( $\mathrm{n}=11$ as determined by T2* losses within the infarct on day 3 CMR; Figure 1) was $40 \%$ lower than that of remote myocardium, suggesting chronic iron deposition $(\mathrm{p}<0.001)$. In contrast, mean T2* of Hemo- infarctions ( $\mathrm{n}=4)$ was not significantly different from that of remote myocardium at both 3 days and 6 months post-angioplasty $(\mathrm{p}=0.51)$.

In canines, in-vivo mean $\mathrm{T} 2 *$ of $\mathrm{Hemo}+$ myocardium was $40 \%$ lower than those of Sham, remote and Hemomyocardium $(\mathrm{p}<0.001)$ at both 3 days and 56 days post-I-R injury (Figure 2B). Similarly, mean ex-vivo $\mathrm{T}^{*}$ of Hemo+ myocardium was $40 \%$ lower than those of Sham, remote

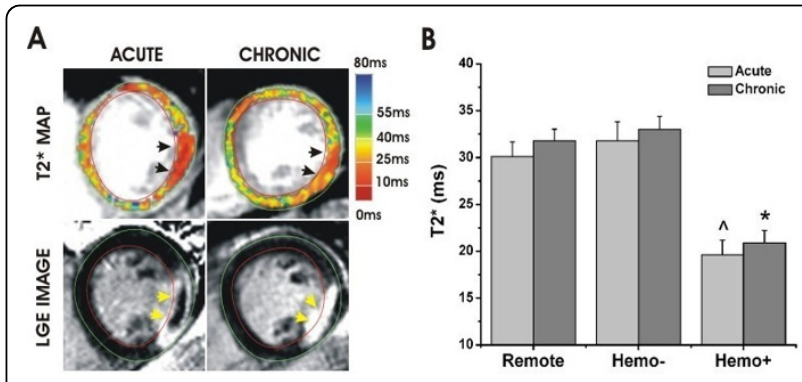

Figure 1 Patient Studies - Representative CMR images (A; acquired from a 42-year old patient following successful angioplasty for first STEMI) with significant T2* loss (arrows) at the site of acute and chronic myocardial infarction (identified by LGE imaging, arrows) are shown. Mean $\mathrm{T}^{*}$ of Hemo+ (B) was $40 \%$ lower than that of Hemoand remote myocardium $(p<0.001)$ on both acute and chronic CMR studies. 


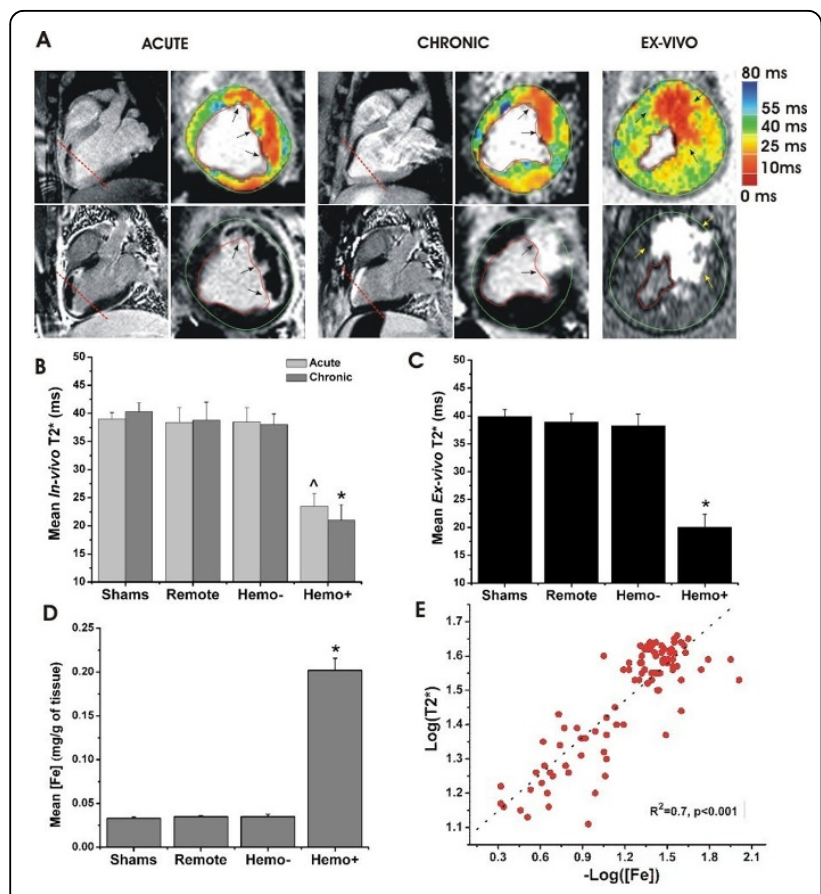

Figure 2 Animal Studies -Representative CMR images (A; T2* in the top row and LGE in the bottom row) acquired from an animal with hemorrhagic myocardial infarction in acute and chronic phases along the long- and short-axis (along the dashed red line in the long-axis images), together with corresponding ex-vivo images are shown (A). In-vivo T2* images (both acute and chronic phases) clearly demonstrate the evidence of signal loss in the LAD territory. Both mean in-vivo T2* (B) and ex-vivo T2* (C) of Hemo+ sections were $40 \%$ lower than those of Shams, Remote, and Hemo- in both acute and chronic phases $(p<0.001)$. Mass spectrometric analysis (D) showed that iron content of Hemo+ tissue was 10-fold higher than that of other tissues $(p<0.001)$. Linear regression analysis $(E)$ between $\log \left(\right.$ ex-vivo $\mathrm{T}^{*}$ ) and $-\log ([\mathrm{Fe}])$ showed a strong correlation $(R 2=0.74 ; p<0.001)$.

and Hemo- myocardium ( $<0.001$; Figure $2 \mathrm{C}$ ). Perl's stain confirmed localized chronic iron deposition only within Hemo+ infarctions. Mean [Fe] of Hemo+ infarctions was nearly 10 -fold higher than those of Sham, remote and Hemo- myocardium ( $<0.001$; Figure 2D). A strong linear relationship was observed between $\log ($ ex-vivo $\mathrm{T} 2 *)$ and $-\log ([\mathrm{Fe}])\left(\mathrm{R}^{2}=0.7 ; \mathrm{p}<0.001\right.$; Figure 2E).

\section{Conclusions}

Acute reperfusion intramyocardial hemorrhage leads to regional chronic iron deposition within the infarct zones. T2* CMR can accurately characterize localized chronic iron deposition following reperfusion-induced myocardial hemorrhage. The clinical significance of this finding remains to be investigated.

\section{Funding}

This work was supported in part by grants from American Heart Association (SDG 0735099N) and National Heart, Lung, And Blood Institute (HL091989).

\section{Author details}

'Biomedical Imaging Research Institute, Cedars-Sinai Medical Center, Los Angeles, CA, USA. ${ }^{2}$ Department of Biomedical Engineering, University of California, Los Angeles, CA, USA. ${ }^{3}$ Quebec Heart \& Lung Institute, Laval University, Quebec City, QC, Canada. ${ }^{4}$ Department of Computer Science and Applications, IMT Institutions, Lucca, Italy. ${ }^{5}$ Montréal Heart Institute, Université de Montréal, Montréal, QC, Canada. ${ }^{6}$ Stephenson CMR Center, University of Calgary, Calgary, AB, Canada.

Published: 30 January 2013

doi:10.1186/1532-429X-15-S1-P174

Cite this article as: Kali et al:: Acute reperfusion intramyocardial

hemorrhage leads to regional chronic iron deposition in the heart. Journal of Cardiovascular Magnetic Resonance 2013 15(Suppl 1):P174.

\section{Submit your next manuscript to BioMed Central} and take full advantage of:

- Convenient online submission

- Thorough peer review

- No space constraints or color figure charges

- Immediate publication on acceptance

- Inclusion in PubMed, CAS, Scopus and Google Scholar

- Research which is freely available for redistribution

Submit your manuscript at www.biomedcentral.com/submit
Biomed Central 